\title{
Simulation numérique du transfert thermique incluant l'ébullition nucléée lors de la coulée continue des métaux
}

\author{
Abdel Illah Nabil Korta ${ }^{\mathrm{a}}$ et Yahia Khadraoui \\ Université de Tlemcen, Faculté des Sciences de l’Ingénieur, Département de Mécanique, BP 230, 13000 Tlemcen, Algérie
}

Reçu le 6 juin 2003, accepté le 8 juillet 2005

\begin{abstract}
Résumé - Un modèle numérique utilisant la méthode de la capacité effective moyenne est présenté pour étudier le transfert thermique dans le processus de solidification lors de la coulée continue avec refroidissement direct. La résolution du problème est faite par la méthode des volumes finis à maillage fixe. Une chaleur spécifique moyenne est donc utilisée pour simuler le volume incluant la zone de changement de phase. Cette méthode surmonte les inconvénients de la méthode de la capacité effective qui est très sensible au choix de l'intervalle de température de changement de phase. Pour déterminer le coefficient de convection à la surface externe des billettes dans la région basse du moule, une méthodologie a été établie en utilisant d'une part, les théories de l'ébullition nucléée avec convection forcée et d'autre part, celles relatives au film de refroidissement. Les résultats de la simulation numérique sont validés par des résultats expérimentaux publiés. L'étude montre que la vitesse de retrait est un facteur très important pour le contrôle de la microstructure et de la condition d'évasion lors de la coulée des métaux.
\end{abstract}

Mots clés : Transfert de chaleur / solidification / coulée continue / volumes finis / ébullition nucléée

\begin{abstract}
A numerical simulation of heat transfer including nucleate boiling during D. C. continuous casting. An algorithm incorporating the effective heat capacity model is described for the finite volume heat transfer analysis involving the two-phase solidification process in direct-chill continuous casting. An average specific heat is employed to simulate the volume including the phase-change zone in this finite volume analysis with fixed mesh. This method overcomes the limitation of the time increments of the otherwise so-called effective heat capacity method. Using theories of nucleate boiling with forced convection and film cooling, a methodology has been devised to evaluate the external boundary condition in the sub mould region of the ingot. The simulation results show very good agreement with the published experimental data. The current study shows that the withdrawal speeds are very important in controlling the microstructure and breakout condition of the cast material.
\end{abstract}

Key words: Heat transfer / solidification / continuous casting / finite volume / nucleate boiling

\section{Introduction}

L'étude thermique des problèmes de changement de phase est d'une grande complexité due à la nature nonlinéaire des équations gouvernantes. Pour la résolution de ce type de problèmes, plusieurs méthodes analytiques ont été développées [1-5]. Cependant, ces techniques sont limitées aux problèmes unidimensionnels car elles aboutissent à des formulations très complexes dans le cas des problèmes multidimensionnels. Les méthodes numériques telles que les différences finies [6], les volumes finis [7] ou les éléments finis [8] semblent être plus pratiques pour la résolution de ces problèmes.

\footnotetext{
a Auteur correspondant : korti72@yahoo.fr
}

Les différents travaux publiés révèlent que ces techniques numériques appliquées aux phénomènes de transfert de chaleur lors du processus de la solidification des métaux peuvent être classées selon deux approches : l'approche à simple région et l'approche à multi-régions. Dans les méthodes à multi-régions, les équations gouvernantes sont appliquées séparément pour chaque phase. La position de l'interface étant inconnue, elle est déterminée en tant qu'élément de la solution. Ces méthodes offrent une bonne précision, mais le coût élevé en termes d'effort de calcul et de stockage d'informations les limite à des problèmes plus simples $[9,10]$. Dans les méthodes à simple région, le maillage reste fixe et l'équation de l'énergie appliquée au liquide et au solide est résolu simultanément en dépit de la discontinuité du gradient de la température à 


\section{Nomenclature}

\begin{tabular}{|c|c|c|}
\hline$C_{\mathrm{p}}$ & chaleur spécifique & $\mathrm{J} \cdot \mathrm{kg}^{-1} \cdot \mathrm{K}^{-1}$ \\
\hline$D$ & diamètre des billettes & $\mathrm{m}$ \\
\hline$g_{0}$ & facteur de conversion & \\
\hline$H_{\text {fg }}$ & chaleur latente d'évaporation & ${\mathrm{J} . \mathrm{kg}^{-1}}^{-1}$ \\
\hline$h_{1}$ & coefficient de convection entre la surface du moule et l'eau de refroidissement primaire & $\mathrm{W} \cdot \mathrm{m}^{2} \cdot \mathrm{K}^{-1}$ \\
\hline$h_{2}(z)$ & coefficient de convection entre la surface des billettes et l'eau de refroidissement secondaire & $\mathrm{W} \cdot \mathrm{m}^{-2} \cdot \mathrm{K}^{-1}$ \\
\hline$h_{\mathrm{c}}, h_{\mathrm{b}}$ & coefficients de convection par convection et par ébullition nucléée & $\mathrm{W} \cdot \mathrm{m}-{ }^{2} \cdot \mathrm{K}^{-1}$ \\
\hline$L$ & hauteur des billettes & $\mathrm{m}$ \\
\hline$L_{1}$ & hauteur du moule & $\mathrm{m}$ \\
\hline$L_{\mathrm{s}}$ & chaleur latente de fusion & $\mathrm{J} \mathrm{kg}^{-1}$ \\
\hline$P$ & pression & $\mathrm{Pa}$ \\
\hline$q / A$ & flux de la chaleur & $\mathrm{W} \cdot \mathrm{m}^{-2}$ \\
\hline$r$ & coordonnée radiale & $\mathrm{m}$ \\
\hline$R_{1}$ & rayon des billettes & $\mathrm{m}$ \\
\hline$R_{2}$ & rayon extérieur du moule & $\mathrm{m}$ \\
\hline$T$ & température & $\mathrm{K}$ \\
\hline$T_{0}$ & température de coulée & $\mathrm{K}$ \\
\hline$T_{\mathrm{f}}$ & température de fusion & $\mathrm{K}$ \\
\hline$T_{\mathrm{f} 1}$ & température de l'eau de refroidissement primaire & $\mathrm{K}$ \\
\hline$T_{\mathrm{f} 2}$ & température de l'eau de refroidissement secondaire & $\mathrm{K}$ \\
\hline$T_{\mathrm{s}}$ & température de la surface externe des billettes & $\mathrm{K}$ \\
\hline$T_{\text {sat }}$ & température de saturation & $\mathrm{K}$ \\
\hline $2 \Delta T$ & intervalle de changement de phase & $\mathrm{K}$ \\
\hline$\Delta T^{\prime}$ & $\begin{array}{l}\text { différence des températures de la surface des billettes et de l'eau de refroidissement } \\
\text { secondaire }\end{array}$ & $\mathrm{K}$ \\
\hline$\Delta T_{x}$ & $\begin{array}{l}\text { différence des températures de la surface des billettes et de saturation de l'eau } \\
\text { de refroidissement }\end{array}$ & $\mathrm{K}$ \\
\hline$u$ & composante axiale de la vitesse & $\mathrm{m} . \mathrm{s}^{-1}$ \\
\hline$v$ & composante radiale de la vitesse & $\mathrm{m} \cdot \mathrm{s}^{-1}$ \\
\hline$U_{\mathrm{s}}$ & vitesse de retrait des billettes & $\mathrm{m} . \mathrm{s}^{-1}$ \\
\hline$z$ & coordonnée axiale & $\mathrm{m}$ \\
\hline \multicolumn{3}{|c|}{ Symboles grecs } \\
\hline$\mu$ & viscosité dynamique & $\mathrm{kg} \cdot \mathrm{m}^{-1} \cdot \mathrm{s}^{-1}$ \\
\hline$\rho$ & densité & $\mathrm{kg} \cdot \mathrm{m}^{-3}$ \\
\hline$\rho_{\mathrm{v}}$ & densité de la vapeur d'eau & $\mathrm{kg} \cdot \mathrm{m}^{-3}$ \\
\hline$\lambda$ & conductivité thermique & $\mathrm{W} \cdot \mathrm{m}^{-1} \cdot \mathrm{K}^{-1}$ \\
\hline$\beta$ & coefficient d'expansion thermique & $\mathrm{K}^{-1}$ \\
\hline$\delta$ & fonction de Dirac & \\
\hline$\sigma$ & tension superficielle & J.m ${ }^{-2}$ \\
\hline \multicolumn{3}{|c|}{ Indices } \\
\hline$\ell$ & liquide ou liquidus & \\
\hline$s$ & solide ou solidus & \\
\hline$e$ & eau & \\
\hline 1 & billette & \\
\hline 2 & moule & \\
\hline
\end{tabular}


un point quelconque de l'interface solide-liquide. L'avantage de ces méthodes réside dans leur simplicité et la facilité avec laquelle elles peuvent être appliquées aux problèmes multidimensionnels. Les méthodes à simple région sont principalement classées en méthodes enthalpiques et méthodes de la capacité effective.

Les méthodes enthalpiques traitent l'enthalpie comme variable dépendant de la température $[11,12]$ alors que les méthodes de la capacité effective introduisent l'effet de la chaleur latente sur la capacité calorifique du matériau $[13,14]$. Pour mieux stabiliser cette dernière, Hsiao expose dans [15] une technique permettant de simuler la zone de changement de phase dans les intervalles limites de températures (zéro et l'infini). Les méthodes de la capacité effective sont simples mais moins précises. Runnels et Carey [16] ont présenté deux arrangements pour améliorer la précision de ces méthodes.

Dans cet article, nous avons intégré la méthode développée par Hsiao [15] afin de stabiliser la méthode de la capacité effective. Elle permet de modéliser les phénomènes de transferts thermiques pendant le processus de coulée continue. L'algorithme utilise une formulation en volumes finis et détermine l'état du matériau à chaque nœud. L'étude des transferts thermiques avec ébullition nucléée à la sortie du moule $[10,17]$ permet de calculer le coefficient de convection à la surface externe des billettes à la sortie du moule.

\section{Modèles et approximations}

\subsection{Modèle physique}

La figure 1 montre schématiquement le processus de la coulée continue des billettes cylindriques en aluminium type A6063 avec refroidissement direct. Le métal liquide à haute température est versé dans le moule qui possède un passage interne à travers lequel circule l'eau de refroidissement primaire. Ce dernier est éjecté vers l'extérieur au bas de moule et empiète directement la surface des billettes. Une enveloppe solide assez résistante pour contenir le métal liquide est ainsi formée, permettant le retrait continu du métal coulé. On distingue alors trois régions : une région solide, une région liquide et une région pâteuse où coexistent les deux phases.

Durant le processus de coulée continue, la distribution de la température des billettes est indépendante du temps et ne dépend que des coordonnées spatiales. Ceci conduit à supposer un transfert thermique stationnaire durant la coulée continue. Vue la symétrie axiale, le système étudié devient bidimensionnel en coordonnées cylindriques. Pour tenir compte du phénomène de changement de phase, nous avons développé un schéma numérique avec maillage fixe utilisant la méthode de la capacité effective moyenne.

\subsection{Modèle d'écoulement}

Le modèle d'écoulement est basé sur les équations de Navier Stokes régissant l'écoulement d'un fluide incompressible, modifiées pour tenir compte des spécificités de

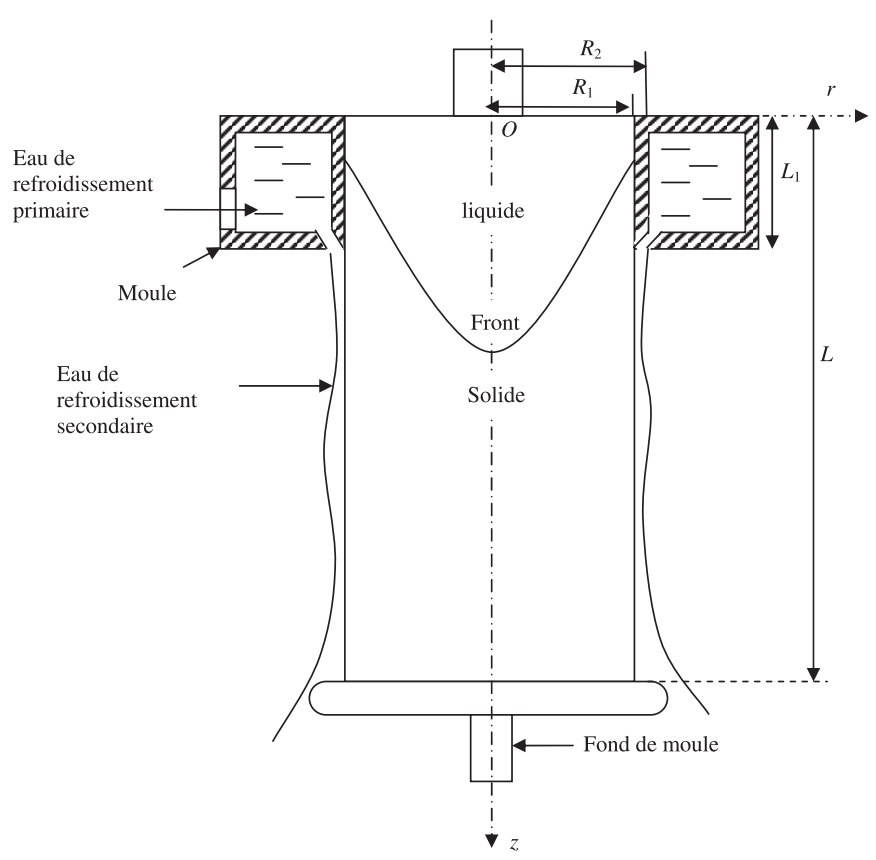

Fig. 1. Diagramme schématique et géométrie de la coulée continue avec refroidissement direct.

changement de phase. Les propriétés thermodynamiques sont indépendantes de la température, sauf dans le terme de la poussée d'Archimède où la variation de la densité $\rho$ engendrée par la température est prise en compte (hypothèse de Boussinesq). On suppose que la phase liquide est un fluide newtonien avec dissipation visqueuse négligeable en écoulement laminaire :

Équation de la conservation de la masse :

$$
\frac{\partial u_{1}}{\partial z}+\frac{1}{r} \frac{\partial\left(r v_{1}\right)}{\partial r}=0
$$

Équations de la conservation de la quantité de mouvement dans les directions $z$ et $r$ :

$$
\begin{aligned}
& \rho_{1} u_{1} \frac{\partial u_{1}}{\partial z}+\rho_{1} v_{1} \frac{\partial u_{1}}{\partial r}= \\
& \frac{\partial}{\partial z}\left(\mu_{1} \frac{\partial u_{1}}{\partial z}\right)+\frac{1}{r} \frac{\partial}{\partial r}\left(r \mu_{1} \frac{\partial u_{1}}{\partial r}\right)-\frac{\partial P_{1}}{\partial z}-\rho_{1} g \beta_{1}\left(T_{1}-T_{\mathrm{f}}\right)
\end{aligned}
$$

$$
\begin{gathered}
\rho_{1} u_{1} \frac{\partial v_{1}}{\partial z}+\rho_{1} v_{1} \frac{\partial v_{1}}{\partial r}= \\
\frac{\partial}{\partial z}\left(\mu_{1} \frac{\partial v_{1}}{\partial z}\right)+\frac{1}{r} \frac{\partial}{\partial r}\left(r \mu_{1} \frac{\partial v_{1}}{\partial r}\right)-\frac{\partial P_{1}}{\partial r}
\end{gathered}
$$

Afin d'éliminer les équations d'écoulement dans la région solide, on choisit de modéliser la viscosité du métal comme fonction de la température. La viscosité dans la région liquide est prise égale à la viscosité du métal liquide, tandis que la viscosité dans la région solide est fixée à une valeur très importante. 


\subsection{Modèle de transfert thermique}

Un modèle basé sur une formulation de la capacité effective moyenne est utilisé pour modéliser le transfert thermique. Les équations gouvernant les problèmes de changement de phase peuvent s'écrire comme suit [10] :

$$
\begin{gathered}
\rho_{1}\left[U_{\mathrm{s}} \frac{\partial H_{1}}{\partial z}\right]=\frac{\partial}{\partial z}\left(\lambda_{\mathrm{s}} \frac{\partial T_{1}}{\partial z}\right)+\frac{1}{r} \frac{\partial}{\partial r}\left(r \lambda_{\mathrm{s}} \frac{\partial T_{1}}{\partial r}\right) \\
\rho_{1}\left[u_{1} \frac{\partial H_{1}}{\partial z}+v_{1} \frac{\partial H_{1}}{\partial r}\right]=\frac{\partial}{\partial z}\left(\lambda_{\ell} \frac{\partial T_{1}}{\partial z}\right)+\frac{1}{r} \frac{\partial}{\partial r}\left(r \lambda_{\ell} \frac{\partial T_{1}}{\partial r}\right)
\end{gathered}
$$

Les deux équations (4) et (5) sont couplées à l'interface solide-liquide en mouvement par les deux équations suivantes :

$$
\begin{gathered}
T_{\mathrm{s}}=T_{\ell}=T_{\mathrm{f}} \\
-\lambda_{\mathrm{s}} \frac{\partial T_{\mathrm{s}}}{\partial n}+\lambda_{\ell} \frac{\partial T_{\ell}}{\partial n}=\rho_{1} L_{\mathrm{s}}(\vec{V} \cdot \vec{n})
\end{gathered}
$$

$\vec{n}$ est le vecteur unitaire normal à l'interface solide-liquide et $\vec{V}$ représente la vitesse de l'interface.

Comme il a été montré dans les travaux [14-16], le système d'équations (4), (5), (6) et (7) peut être réduit à une seule équation :

$$
\rho_{1}\left[u_{1} \frac{\partial H_{1}}{\partial z}+v_{1} \frac{\partial H_{1}}{\partial r}\right]=\frac{\partial}{\partial z}\left(\lambda_{1} \frac{\partial T_{1}}{\partial z}\right)+\frac{1}{r} \frac{\partial}{\partial r}\left(r \lambda_{1} \frac{\partial T_{1}}{\partial r}\right)
$$

avec

$$
\lambda_{1}=\left\{\begin{array}{lll}
\lambda_{\mathrm{s}} & \text { si } & T_{1}<T_{\mathrm{f}} \\
\lambda_{\ell} & \text { si } & T_{1}>T_{\mathrm{f}}
\end{array}\right.
$$

L'enthalpie totale d'un métal subissant un changement de phase à la température $T_{\mathrm{f}}$ est définie par :

$$
H_{1}\left(T_{1}\right)=\int_{T_{\mathrm{r}}}^{T_{1}}\left[C_{p 1}\left(T_{1}\right)+L_{\mathrm{s}} \eta\left(T_{1}-T_{\mathrm{f}}\right)\right] \mathrm{d} T_{1}
$$

avec

$$
\eta\left(T_{1}-T_{\mathrm{f}}\right)=\left\{\begin{array}{lll}
1 & \text { si } & T_{1}-T_{\mathrm{f}} \geq 0 \\
0 & \text { si } & T_{1}-T_{\mathrm{f}}<0
\end{array}\right.
$$

où $T_{\mathrm{r}}<T_{\mathrm{f}}$ est une température arbitrairement choisie. La capacité effective $C_{\text {eff }}\left(T_{1}\right)$ peut être définie alors comme suit :

$$
C_{\text {eff }}\left(T_{1}\right)=\frac{\mathrm{d} H_{1}\left(T_{1}\right)}{\mathrm{d} T_{1}}=C_{p 1}\left(T_{1}\right)+L_{\mathrm{s}} \delta\left(T_{1}-T_{\mathrm{f}}\right)
$$

avec

$$
C_{p 1}\left(T_{1}\right)=\left\{\begin{array}{lll}
C_{p s}\left(T_{1}\right) & \text { si } & T<T_{\mathrm{f}} \\
C_{p \ell}\left(T_{1}\right) & \text { si } & T>T_{\mathrm{f}}
\end{array}\right.
$$

$\delta\left(T_{1}-T_{\mathrm{f}}\right)$ représente la fonction de Dirac qui est infinie à $T_{1}=T_{\mathrm{f}}$ et nulle ailleurs. Elle satisfait l'équation suivante :

$$
\int_{-\infty}^{+\infty} \delta\left(T_{1}-T_{\mathrm{f}}\right) \mathrm{d} T_{1}=1
$$

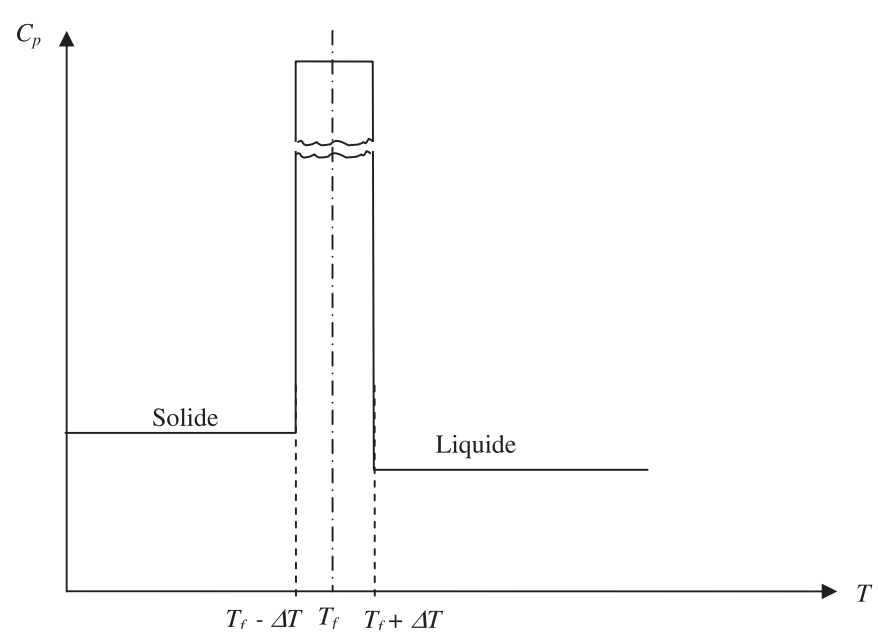

Fig. 2. Chaleur spécifique combinée incluant l'effet de la chaleur latente dans l'intervalle $2 \Delta T$ au voisinage de la température de fusion.

En remplaçant les équations (10a) et (10b) dans l'équation (8a), on trouve l'expression suivante :

$$
\begin{aligned}
\rho_{1} C_{\text {eff }}\left[u_{1} \frac{\partial T_{1}}{\partial z}+v_{1} \frac{\partial T_{1}}{\partial r}\right] & = \\
& \frac{\partial}{\partial z}\left(\lambda_{1} \frac{\partial T_{1}}{\partial z}\right)+\frac{1}{r} \frac{\partial}{\partial r}\left(r \lambda_{1} \frac{\partial T_{1}}{\partial r}\right)
\end{aligned}
$$

Puisque, nous ne pouvons jamais avoir strictement $T_{1}=T_{\mathrm{f}}$, la fonction de Dirac est remplacée par la fonction delta qui attribue une valeur importante à la chaleur spécifique dans un petit intervalle de changement de phase $\left(T_{\mathrm{f}}-\Delta T / 2\right)$ à $\left(T_{\mathrm{f}}+\Delta T / 2\right)$ (Fig. 2). En supposant que $C_{\mathrm{ps}}, C_{\mathrm{p} \ell}, \lambda_{\mathrm{s}}$ et $\lambda_{\ell}$ sont constantes, les équations (10a), (10b) et $(8 \mathrm{~b})$ peuvent être approximées comme suit :

$C_{\mathrm{eff}}= \begin{cases}C_{\mathrm{ps}} & \text { si } T_{1}<T_{\mathrm{f}}-\Delta T \\ \frac{C_{\mathrm{p} \ell}+C_{\mathrm{ps}}}{2}+\frac{L_{\mathrm{s}}}{2 \Delta T} & \text { si } T_{\mathrm{f}}-\Delta T \leq T_{1} \leq T_{\mathrm{f}}+\Delta T \\ C_{\mathrm{p} \ell} & \text { si } T_{1}>T_{\mathrm{f}}+\Delta T\end{cases}$

$$
\lambda_{1}=\left\{\begin{array}{lll}
\lambda_{\mathrm{s}} & \text { si } & T_{1}<T_{\mathrm{f}}-\Delta T \\
\frac{\lambda_{\ell}+\lambda_{\mathrm{s}}}{2} & \text { si } & T_{\mathrm{f}}-\Delta T \leq T_{1} \leq T_{\mathrm{f}}+\Delta T \\
\lambda_{\ell} & \text { si } & T_{1}>T_{\mathrm{f}}+\Delta T
\end{array}\right.
$$

Les algorithmes utilisant l'équation (12) sont sensibles à la valeur de l'intervalle de température $2 \Delta T$ sélectionné. D'une part, l'intervalle $2 \Delta T$ doit avoir une valeur minimale pour simuler les métaux purs et d'autre part, il doit avoir une valeur importante afin de réduire la non-linéarité provoquée par l'effet de la chaleur latente $\frac{L_{\mathrm{s}}}{2 \Delta T}$ et de simuler les alliages. Pour stabiliser la solution, Hsiao [15] suggère l'arrangement suivant : au lieu d'utiliser directement, dans l'équation (12), la température nodale pour déterminer la chaleur spécifique correspondante 
du nœud, il prend une chaleur spécifique moyenne dont la valeur est tirée de la température au nœud $(i, j)$ et des nœuds adjacents :

$$
\begin{aligned}
C_{\text {eff }}\left(T_{i, j}\right)= & \frac{1}{4}\left[C_{\text {eff }}\left(T_{i, j}, T_{i-1, j}\right)+C_{\text {eff }}\left(T_{i, j}, T_{i+1, j}\right)\right. \\
& \left.+C_{\text {eff }}\left(T_{i, j}, T_{i, j-1}\right)+C_{\text {eff }}\left(T_{i, j}, T_{i, j+1}\right)\right]
\end{aligned}
$$

où $T_{i, j}$ représente la température au nœud $(i, j)$.

\section{Conditions aux limites}

Les conditions aux limites associées au problème sont formulées comme suit :

- pour $z=0$ et $0 \leq r \leq R_{1}$ :

$$
\begin{aligned}
u_{1}(r, 0) & =U_{\mathrm{s}} \\
v_{1}(r, 0) & =0 \\
T_{1}(r, 0) & =T_{0}
\end{aligned}
$$

- pour $z=L$ et $0 \leq r \leq R_{1}$ :

$$
\begin{aligned}
u_{1}(r, L) & =U_{\mathrm{s}} \\
v_{1}(r, L) & =0 \\
-\lambda_{1} \frac{\partial T_{1}}{\partial z}(r, L) & =\rho_{1} C_{\mathrm{ps}} U_{\mathrm{s}} T_{1}(r, L)
\end{aligned}
$$

- pour $r=0$ et $0 \leq z \leq L$ :

$$
\begin{array}{r}
\frac{\partial u_{1}}{\partial r}(0, z)=0 \\
v_{1}(0, z)=0 \\
\frac{\partial T_{1}}{\partial r}(0, z)=0
\end{array}
$$

- pour $r=R_{1}$ et $0 \leq z \leq L$ :

$$
\begin{aligned}
& u_{1}\left(R_{1}, z\right)=U_{\mathrm{s}} \\
& v_{1}\left(R_{1}, z\right)=0
\end{aligned}
$$

pour $0 \leq z \leq L_{1}$ (interface métal-moule) :

$$
\begin{aligned}
\lambda_{1} \frac{\partial T_{1}}{\partial r}\left(R_{1}, z\right) & =\lambda_{2} \frac{\partial T_{2}}{\partial r}\left(R_{1}, z\right) \\
T_{1} & =T_{2}
\end{aligned}
$$

pour $L_{1} \leq z \leq L$ :

$$
-\lambda_{1} \frac{\partial T_{1}}{\partial r}\left(R_{1}, z\right)=h_{2}(z) \cdot\left[T_{1}\left(R_{1}, z\right)-T_{f 2}\right]
$$

- pour $R_{1}<r<R_{2}$ et $0 \leq z \leq L_{1}$ (transfert de chaleur par conduction dans le moule) :

$$
\frac{\partial}{\partial z}\left(\lambda_{2} \frac{\partial T_{2}}{\partial z}\right)+\frac{1}{r} \frac{\partial}{\partial r}\left(r \lambda_{2} \frac{\partial T_{2}}{\partial r}\right)=0
$$

- pour $r=R_{2}$ et $0 \leq z \leq L_{1}$ :

$$
-\lambda_{2} \frac{\partial T_{2}}{\partial r}\left(R_{2}, z\right)=h_{1}\left[T_{2}\left(R_{2}, z\right)-T_{f 1}\right]
$$

Une approximation différences finies est utilisée pour approcher les différentes conditions aux limites.

\section{Transfert de chaleur avec ébullition nucléée}

Une méthode de calcul du coefficient de convection dans la région basse du moule des billettes refroidies par une chute libre d'eau a été développée par Weckman [18]. Elle utilise les théories existantes de l'ébullition nucléée avec convection forcée et les théories relatives au film de refroidissement. Pour un cylindre refroidi avec un film d'eau, le nombre de Reynolds est défini par l'équation suivante :

$$
R e=\frac{4 \rho_{\mathrm{e}} Q}{\pi D \mu_{\mathrm{e}}}
$$

Les billettes ont un diamètre de $0,1524 \mathrm{~m}$, la température initiale de l'eau est de $283 \mathrm{~K}$ et le débit de l'eau de refroidissement $Q$ est égal à $0,00189 \mathrm{~m}^{3} \cdot \mathrm{s}^{-1}$. En remplaçant ces valeurs dans l'équation (21), le nombre de Reynolds aura la valeur 12000 et le régime d'écoulement est turbulent.

Le coefficient de convection dépend fortement de la température moyenne de l'eau $\bar{T}$ définie comme suit :

$$
\bar{T}=\frac{T_{\mathrm{s}}+T_{f 2}}{2}
$$

Une analyse de régression linéaire de $h_{\mathrm{c}}$ en fonction de $\bar{T}$ donne l'équation dimensionnelle suivante $[10,18]$

$$
h_{\mathrm{c}}=\left[-1,67 \times 10^{5}+704 \bar{T}\right]\left(\frac{Q}{\pi D}\right)^{1 / 3}
$$

La chaleur dégagée par les billettes est donnée par l'équation dimensionnelle suivante

$$
\left(\frac{q}{A}\right)_{\mathrm{c}}=\left[-1,67 \times 10^{5}+704 \bar{T}\right]\left(\frac{Q}{\pi D}\right)^{1 / 3} \Delta T^{\prime}
$$

Une fois le régime d'ébullition nucléée atteint, la chaleur évacuée des billettes sera contrôlée par $\Delta T_{x}$ au lieu de $\Delta T^{\prime}$.

Le flux de chaleur donné par ébullition en eau stagnante est déduit à partir de la corrélation suivante [19,20]

$$
\frac{c_{\mathrm{pe}} \Delta T_{x}}{H_{\mathrm{fg}}}=C_{\mathrm{f}}\left[\frac{\left(\frac{q}{A}\right)_{\mathrm{b}}}{\mu_{\mathrm{e}} H_{\mathrm{fg}}} \sqrt{\frac{g_{0} \sigma}{g\left(\rho_{\mathrm{e}}-\rho_{v}\right)}}\right]^{0,33}\left[\frac{c_{\mathrm{pe}} \mu_{\mathrm{e}}}{\lambda_{\mathrm{e}}}\right]^{1,7}
$$

Le coefficient $g_{0}$ est un facteur de conversion égal à l'unité dans le système international des unités (SI). Le coefficient $C_{\mathrm{f}}$ est égal à 0,016 pour l'aluminium type A6063 refroidi par une chute libre d'eau [10]. Les propriétés physiques de l'eau de refroidissement dans l'équation (25) correspondent à la température de saturation $\left(100{ }^{\circ} \mathrm{C}\right.$ à la pression atmosphérique). En remplaçant ces paramètres par leurs valeurs, l'équation (25) prend la forme simple suivante :

$$
\left(\frac{q}{A}\right)_{\mathrm{b}}=20,8\left(\Delta T_{x}\right)^{3}
$$

Pour étudier les problèmes combinés de la convection forcée avec ébullition nucléée, Rohsenow recommande 
d'ajouter le flux de chaleur dû à la convection forcée au flux de chaleur provenant de l'ébullition en eau stagnante $[19,20]$ :

$$
\left(\frac{q}{A}\right)_{\text {total }}=\left(\frac{q}{A}\right)_{\mathrm{c}}+\left(\frac{q}{A}\right)_{\mathrm{b}}
$$

Des équations (24), (26) et (27) on déduit l'équation suivante :

$$
\begin{array}{r}
\left(\frac{q}{A}\right)_{\text {total }}=\left(-1,67 \times 10^{5}+704 \bar{T}\right)( \\
\left.\frac{Q}{\pi D}\right)^{1 / 3} \Delta T \\
+20,8\left(\Delta T_{x}\right)^{3}
\end{array}
$$

Le coefficient de transfert thermique dans la zone d'ébullition nucléée est alors :

$h_{2}(z)=\left(-1,67 \times 10^{5}+704 \bar{T}\right)\left(\frac{Q}{\pi D}\right)^{1 / 3}+\frac{20,8}{\Delta T}\left(\Delta T_{x}\right)^{3}$

Le point de naissance de l'ébullition nucléée est obtenu à partir des travaux de Weckman $[19,20]$

$$
\left(\frac{q}{A}\right)_{i}=3910\left(\Delta T_{x}\right)^{2,16}
$$

Les deux équations (29) et (30) permettent de calculer le coefficient de convection $h_{2}(z)$. La température $\left(\Delta T_{x}\right)$ est calculée à partir des résultats expérimentaux [18].

\section{Procédure numérique}

La discrétisation numérique des équations de transport a été effectuée par la méthode des volumes finis et ont été résolues en utilisant l'algorithme $\boldsymbol{S I M P L E}$. Pour intégrer le modèle de la capacité effective dans la formulation volumes finis, nous avons utilisé la méthode de la capacité calorifique moyenne développée par Hsiao [27].

Les maillages de $150 \times 70$ éléments dans les billettes et $50 \times 30$ éléments dans le moule ont été choisis pour simuler la géométrie étudiée.

Une méthode implicite est adoptée pour la discrétisation des équations de continuité, de quantité de mouvement et de l'énergie. La méthode semi-itérative ligne par ligne est utilisée pour obtenir la solution. Pour les différentes vitesses de coulée, les facteurs de sous-relaxation qui semblent favoriser la stabilité du processus itératif sont : 0,46 pour les deux composantes de la vitesse $u_{1}$ et $v_{1}$ et 0,8 pour $T_{1}$. Les itérations sont arrêtées lorsque tous les résidus absolus sont inférieurs à $10^{-4}$.

Lorsque la température atteint la température de fusion $T_{\mathrm{f}}$, le vecteur vitesse dans l'équation de l'énergie est modifié. La composante axiale $u_{1}$ est fixée à la vitesse de la coulée et la composante radiale $v_{1}$ est fixée à zéro.

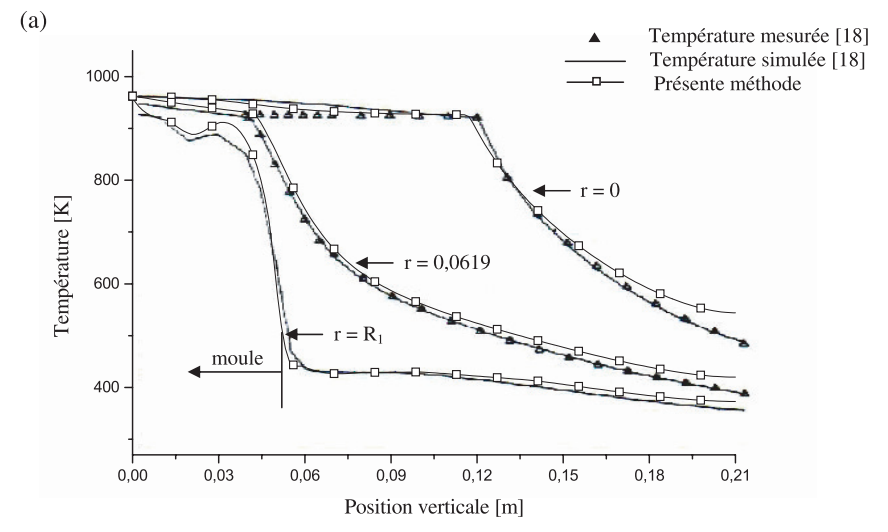

(b)

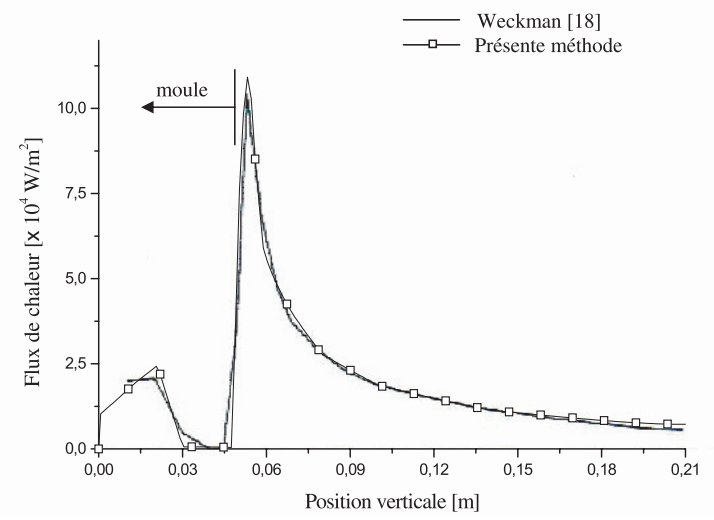

Fig. 3. Comparaison entre les résultats expérimentaux et les résultats calculés.

\section{Résultats et discussion}

La simulation numérique de la coulée continue avec refroidissement direct, a été effectuée pour des billettes cylindriques en alliage $\mathrm{Al}-\mathrm{Mg}$ type $\mathrm{A} 6063$ de diamètre $0,1524 \mathrm{~m}$. Le moule est fabriqué par le même type d'alliage. Les propriétés thermophysiques sont supposées constantes pour chaque phase.

Pour l'application numérique, nous avons pris les valeurs suivantes : $\lambda_{\mathrm{s}}=210 \mathrm{~W} \cdot \mathrm{m}^{-1} \cdot \mathrm{K}^{-1}, \quad \lambda_{\ell}=$ $95 \mathrm{~W} \cdot \mathrm{m}^{-1} \cdot \mathrm{K}^{-1}, \quad c_{\mathrm{ps}}=1120 \mathrm{~J} \cdot \mathrm{kg}^{-1} \cdot \mathrm{K}^{-1}, \quad c_{\mathrm{p} \ell}=$ $1075 \mathrm{~J} \mathrm{~kg}^{-1} \cdot \mathrm{K}^{-1}, \rho=2700 \mathrm{~kg} \cdot \mathrm{m}^{-3}, T_{0}=962 \mathrm{~K}, T_{\mathrm{f}}=$ $923 \mathrm{~K}, L_{\mathrm{s}}=386786,4 \mathrm{~J}_{\mathrm{kg}}{ }^{-1}, T_{\mathrm{f} 1}=282 \mathrm{~K}$ et $T_{\mathrm{f} 2}=302 \mathrm{~K}$.

Les résultats obtenus par la simulation numérique ont été comparés avec ceux obtenus par Weckman [18]. Un test sur le choix de l'intervalle de solidification $(2 \Delta T)$ qui assure la meilleure précision a été effectué d'abord. Un intervalle de $6 \mathrm{~K}$ semble accomplir cette tâche. Les figures $3 \mathrm{a}$ et $3 \mathrm{~b}$ présentent l'évolution des températures calculées à la surface externe, pour $r=0,0619 \mathrm{~m}$ et au niveau de l'axe, ainsi que le flux de chaleur à la surface pour des billettes coulées à une vitesse de retrait de $0,00381 \mathrm{~m} \cdot \mathrm{s}^{-1}$. Les résultats obtenus sont en bon accord avec ceux obtenus expérimentalement et numériquement dans les travaux [18]. Les résultats numériques de la référence [18] ont été obtenus par la méthode multi-régions qui est considérée comme la plus précise des méthodes utilisées pour la résolution des problèmes de changement de phase. 


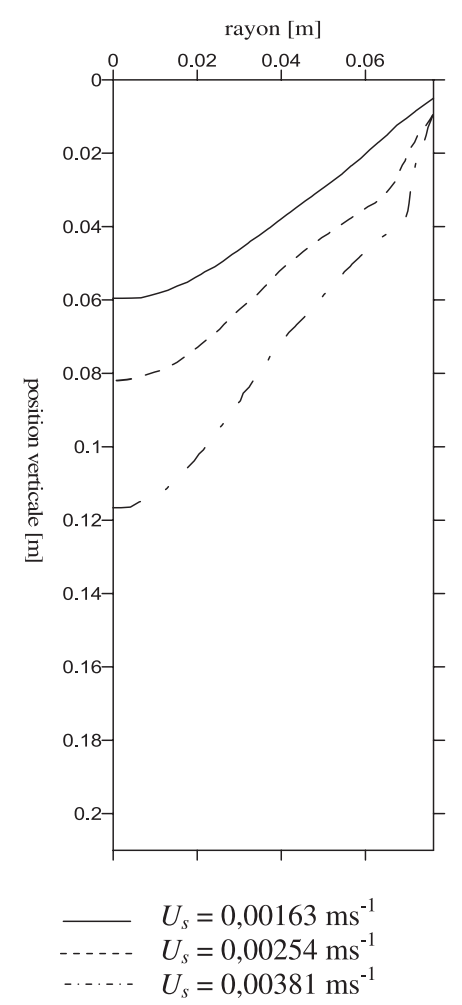

Fig. 4. Effet de la vitesse de retrait.

Ceci souligne la bonne précision de la méthode utilisée dans la présente étude.

La figure 4 montre l'effet de la vitesse de retrait sur la position et la forme du front de solidification. Nous observons qu'avec l'augmentation de la vitesse de retrait, le front de solidification se déplace vers le bas. Ceci permet de prévoir une vitesse optimale de la coulée en évitant la condition d'évasion du métal liquide à la sortie du moule. Avec l'augmentation de la vitesse de retrait, la vitesse du métal coulé augmente et le temps nécessaire pour extraire la chaleur dans la région du moule s'affaiblit.

La figure 5 montre les profils de la température calculée (isothermes) pour des billettes coulées à des différentes vitesses de retrait. On peut facilement constater qu'avec l'augmentation de la vitesse de retrait, la température de la surface extérieure des billettes devient plus importante. Quand cette température dépasse la température critique, il y a risque de formation aléatoire d'un film de vapeur qui isole localement et momentanément les billettes de l'eau de refroidissement et, les degrés d'extraction de la chaleur deviennent faibles. Si les degrés d'extraction de la chaleur par le film bouillant sont insuffisants, l'évasion du métal liquide peut se produire. Pour les grandes vitesses de retrait, nous remarquons qu'il y a un risque de réchauffement des billettes à l'intérieur du moule qui peut entraîner la fusion de l'enveloppe solidifiée et donc la rupture des billettes.

La figure 6 montre l'effet de la vitesse de retrait sur le champ de vitesse calculé. Le champ de vitesse permet d'identifier le chemin parcouru par les particules lors de l'écoulement. Pour une faible vitesse de retrait
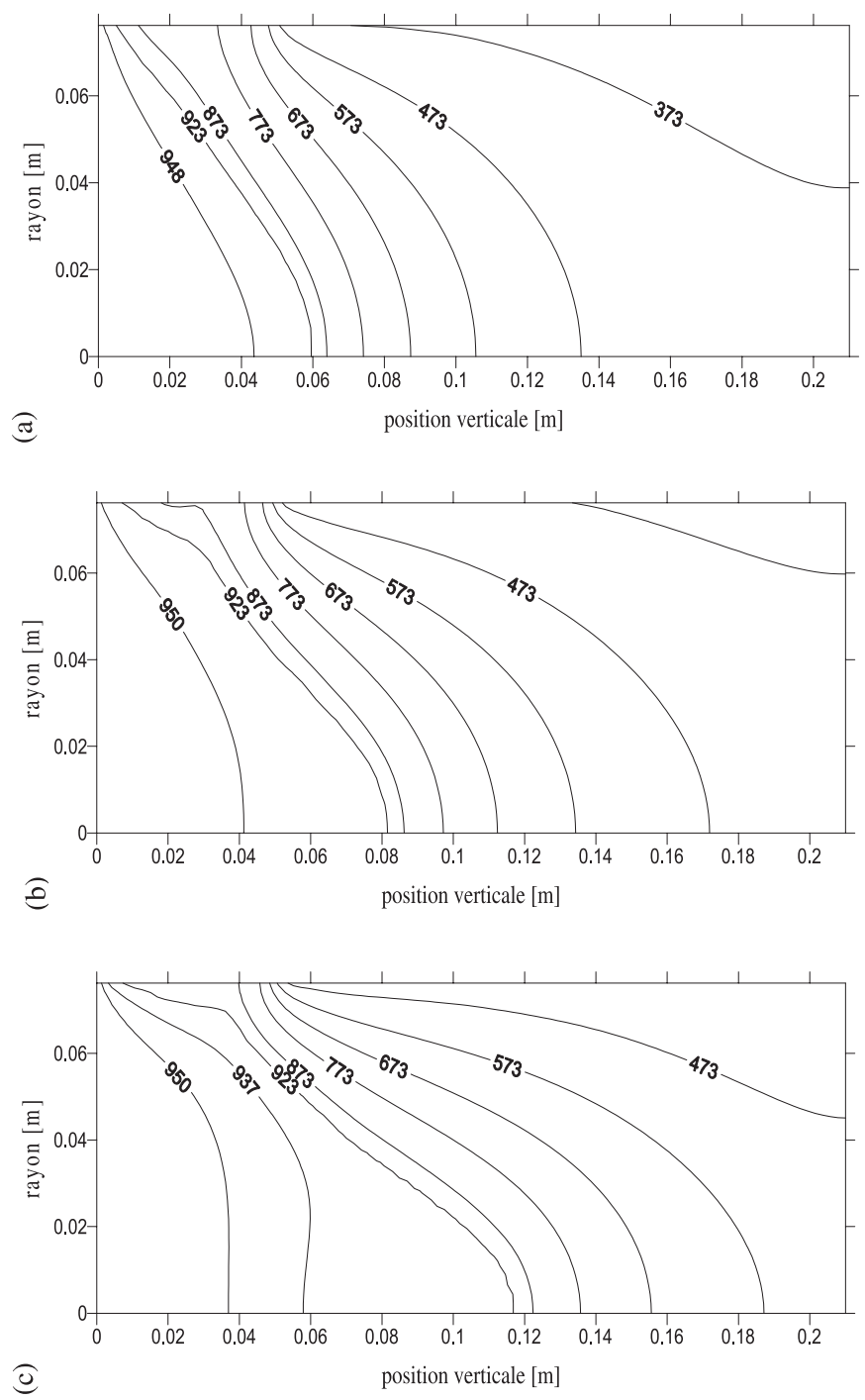

Fig. 5. Positions de l'interface solide-liquide et profil de la température (isothermes) pour des vitesses de retrait (a) $0,00163 \mathrm{~m} \cdot \mathrm{s}^{-1}$, (b) $0,00254 \mathrm{~m} \cdot \mathrm{s}^{-1}$ et (c) $0,00381 \mathrm{~m} \cdot \mathrm{s}^{-1}$.

$\left(1,63 \times 10^{-3} \mathrm{~m} \cdot \mathrm{s}^{-1}\right)$, le métal liquide stagnant pénètre à une profondeur de $0,059 \mathrm{~m}$, tandis que pour une vitesse de coulée de $2,54 \times 10^{-3} \mathrm{~m} . \mathrm{s}^{-1}$, la profondeur est de $0,081 \mathrm{~m}$. Pour une grande vitesse de coulée $\left(3,81 \times 10^{-3} \mathrm{~m} . \mathrm{s}^{-1}\right)$, le métal liquide stagnant pénètre à une profondeur de 0,117 m. L'épaisseur de l'enveloppe solidifiée augmente avec la direction axiale et au bas du moule, elle est de $0,051,0,035$ et $0,02 \mathrm{~m}$. Ces épaisseurs correspondent à $67 \%, 46 \%$ et $26 \%$ du rayon des billettes pour les vitesses de coulée de $1,63 \times 10^{-3}, 2,54 \times 10^{-3}$ et $3,81 \times 10^{-3} \mathrm{~m} . \mathrm{s}^{-1}$, respectivement. Pour les grandes vitesses de retrait, nous remarquons la naissance d'un mouvement circulaire dans la phase liquide qui marque l'importance du transfert de chaleur convectif dans cette région.

La figure 7 montre l'évolution du flux de chaleur à la surface des billettes coulées à différentes vitesses de retrait. On peut voir qu'avec l'augmentation de la vitesse de retrait, le flux de chaleur à la surface augmente. Avec 

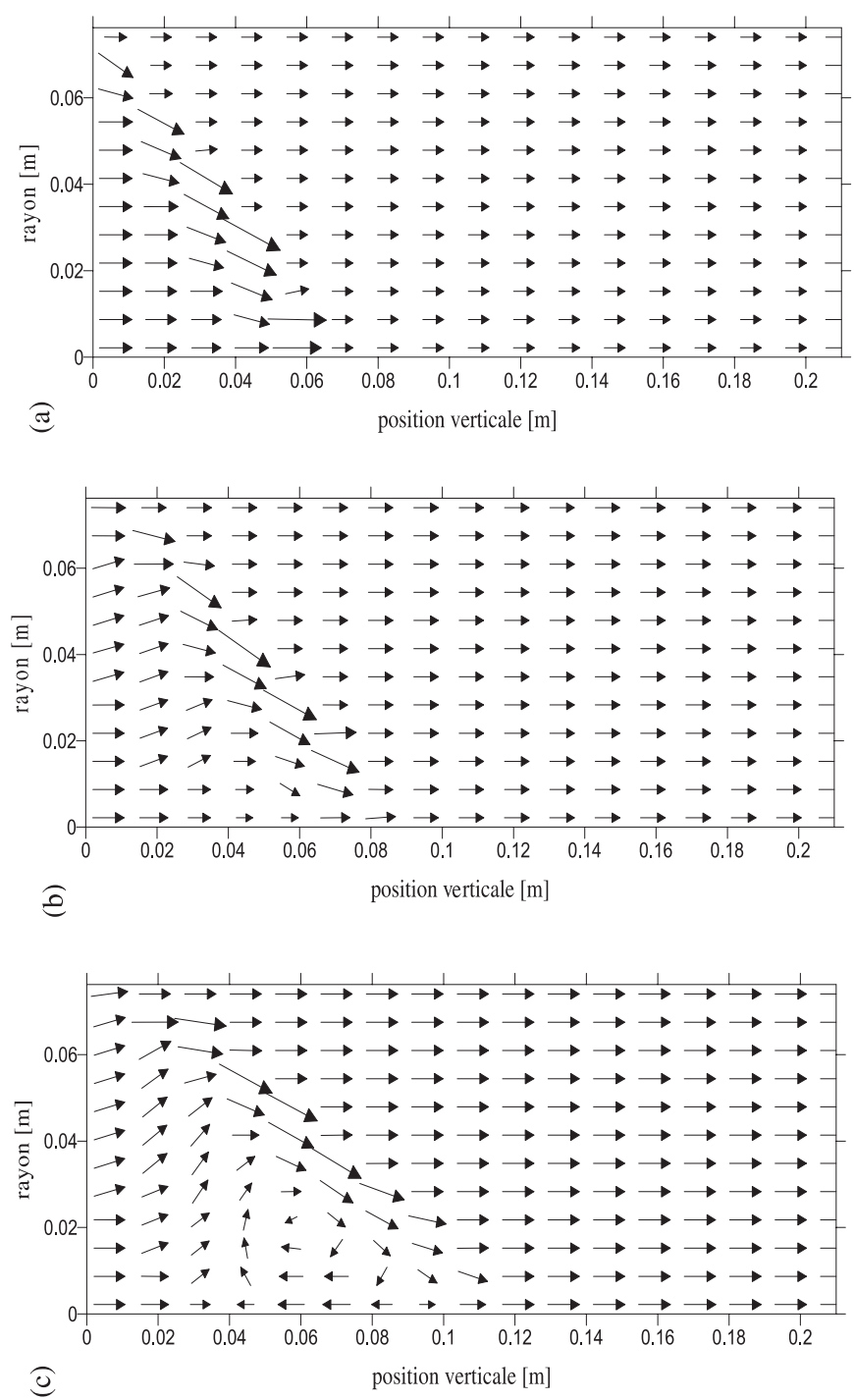

Fig. 6. Effet de la vitesse de retrait sur le champ de vitesse (a) $0,00163 \mathrm{~m} \cdot \mathrm{s}^{-1}$, (b) $0,00254 \mathrm{~m} \cdot \mathrm{s}^{-1}$ et (c) $0,00381 \mathrm{~m} \cdot \mathrm{s}^{-1}$.

l'augmentation de la vitesse, la quantité du métal coulé augmente d'où une quantité de chaleur importante à extraire. La discontinuité de la courbe est due au changement des conditions de refroidissement (région moule et région basse de moule).

Les figures $8 \mathrm{a}$ et $8 \mathrm{~b}$ montrent l'évolution de la température au niveau de l'axe et de la surface extérieure des billettes coulées à des vitesses différentes de retrait. On constate qu'avec l'augmentation de la vitesse de retrait, la température de la surface extérieure des billettes prend des valeurs plus élevées.

Les paliers quasi-constants des températures dans la figure 8a (dans la direction $z$ ) correspondent aux zones qui marquent le début et la fin de la solidification (Fig. 4). Cela montre que la température reste constante dans la région du changement de phase.

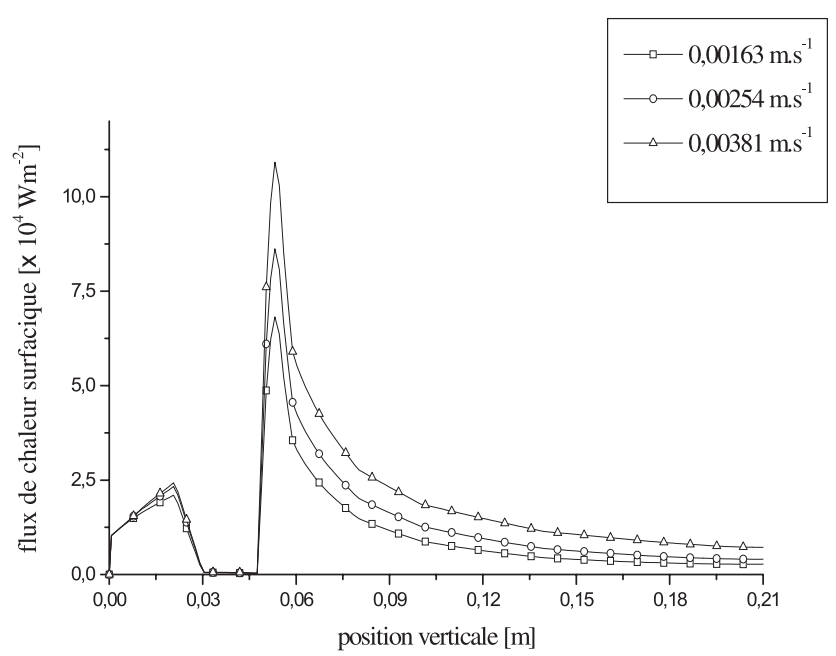

Fig. 7. Effet de la vitesse de retrait sur le flux de chaleur à la surface des billettes.

(a)
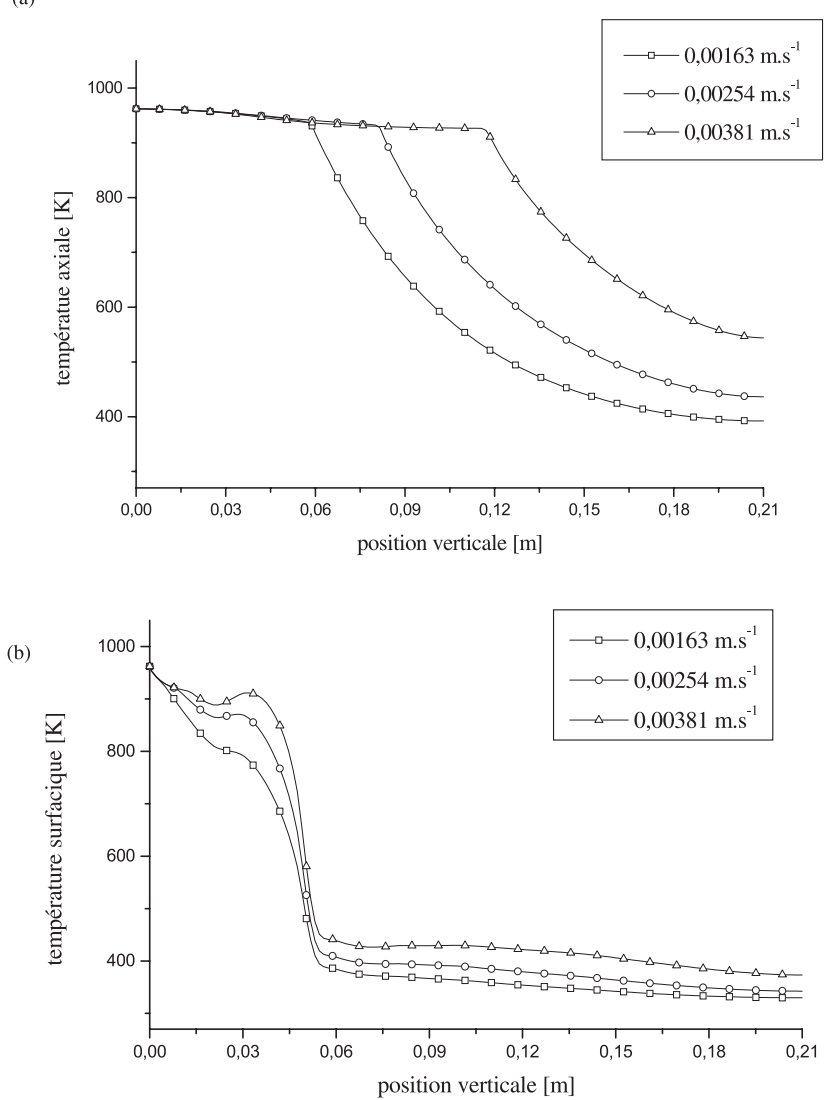

Fig. 8. Effet de la vitesse de retrait sur (a) la température à l'axe (b) la température à la surface extérieure.

\section{Conclusion}

La méthode de la capacité effective moyenne résolue par la méthode des volumes finis a été employée pour résoudre le processus de la solidification lors de la coulée continue avec refroidissement direct. Une bonne concordance avec les résultats expérimentaux a été obtenue. 
Dans ce travail, les théories de l'ébullition nucléée avec convection forcée et celles relatives au film de refroidissement ont été utilisées pour déterminer le coefficient de convection à la surface externe des billettes dans la région basse du moule. La vitesse de retrait a un effet très significatif sur le processus de la solidification. Elle permet de contrôler la position et la forme du front de solidification et permet aussi de déterminer la condition d'évasion du métal liquide. Avec l'augmentation de la vitesse de retrait, la température de la surface extérieure des billettes devient plus importante. Il y a donc, risque de formation aléatoire d'un film de vapeur qui diminue les taux d'extraction de la chaleur et l'évasion du métal liquide peut se produire.

\section{Références}

[1] T.R. Goodman, The heat balance integral and its application to problems involving a change of phase, Trans. ASME 80 (1958) 353-342

[2] L.T. Yeh, B.T.F. Chung, Solidification and melting of material subjected to convection and radiation, J. Spacer. Rockets 12 (1975) 329-334

[3] B.A. Boley, The embedding technique in melting and solidification problems, moving boundary problems in heat flow and diffusion, in J. Ockendon, W. Hodgkins, ed., Proceedings of the conferences held at the University of Oxford, 1974, pp. 150-172

[4] J. Crank, R. Gupta, Isotherm migration method in two dimensions, Int. J. Heat Mass Transfer 18 (1975) 1101-1117

[5] C.S. Keung, The Use of sources and sinks in solving twodimensional heat conduction problems with change of phase in arbitrary domains, $\mathrm{PhD}$ dissertation, Columbia Univ. New York, 1980

[6] A. Lazarids, A numerical solution of multidimensional solidification (or melting) problems, Int. Heat Mass Transfer 13 (1970) 1459-1477

[7] X.K. Lan, J.M. Khodadadi, Fluid flow, heat transfer and solidification in the mold of continuous casters during ladle change, Int. J. heat and mass Tran. 44 (2001) 953-965
[8] J.S. Hsiao, B.T.F. Chung, An efficient algorithm for finite element solution to two-dimensional heat transfer with melting and freezing, ASME paper 84-HT-2, presented at the $22 \mathrm{~d}$ national heat transfer Conf., Niagara Falls, 1984

[9] D. Lynch, K. O'Neil, Continuously deforming finite elements for the solution of parabolic problems with and without phase change, Int. J. Numer. Meth. Eng. 17 (1981) $81-96$

[10] S.K. Das, Thermal modelling of D. C. continuous casting including sub-mould boiling heat transfer, Applied Thermal Engineering 19 (1999) 897-916

[11] N. Shamsundar, E. Sparrow, Analysis of multidimensional phase change via the enthalpy model, J. Heat Transfer, Trans. ASME 19 (1975) 333-340

[12] G. Comini, S. Guidice, R. Del Lewis, O. Zienkiewicz, Finite element solution of non-linear heat conduction problems with special reference to phase change, Int. J. Numer. Methods Eng. 8 (1974) 613-624

[13] M. Ruhul Amin, D. Greif, Conjugate heat transfer during two-phase solidification process in a continuously moving metal using average heat capacity method, Int. J. Heat Trans. 42 (1999) 2883-2895

[14] R.T. Lee, W.Y. Chiou, Finite element analysis of phase change problems using multilevel techniques, Numer. Heat Trans. Part B 27 (1995) 277-290

[15] J.S. Hsiao, An efficient algorithm for finite difference analyses of heat transfer with melting and solidification, Numer. Heat Trans. 8 (1985) 653-666

[16] S.R. Runnels, G.F. Carey, Finite element simulation of phase change using capacitance methods, Numer. Heat Trans. 19 (1991) 13-30

[17] Y. Khadraoui, A.N. Korti, Z. Senouci, F.Y. TabetHellal, M.A. Ghernaout, Simulation numérique du transfert thermique dans un métal en voie de solidification, J. Maghrébin de Physique 1 (2000) 57-60

[18] D.C. Weckman, P. Niessen, A numerical simulation of the D. C. continuous casting process including nucleate boiling heat transfer, Metall. Trans. 13B (1982) 593-602

[19] W.M. Rohsenow, Developments in heat transfer, MIT Press, Cambridge, 1964

[20] W.M. Rohsenow, Modern developments in heat transfer, Academic Press, New York, 1963

Retrouvez nos articles sur le site : www.edpsciences.org/meca 